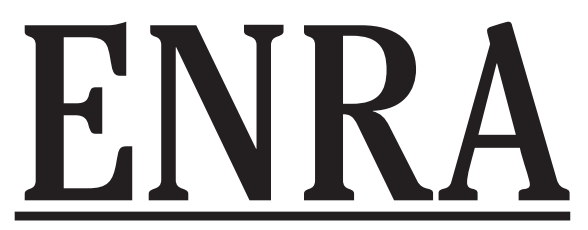

Q U A D E R N S

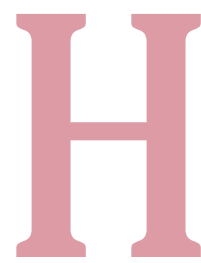

D E
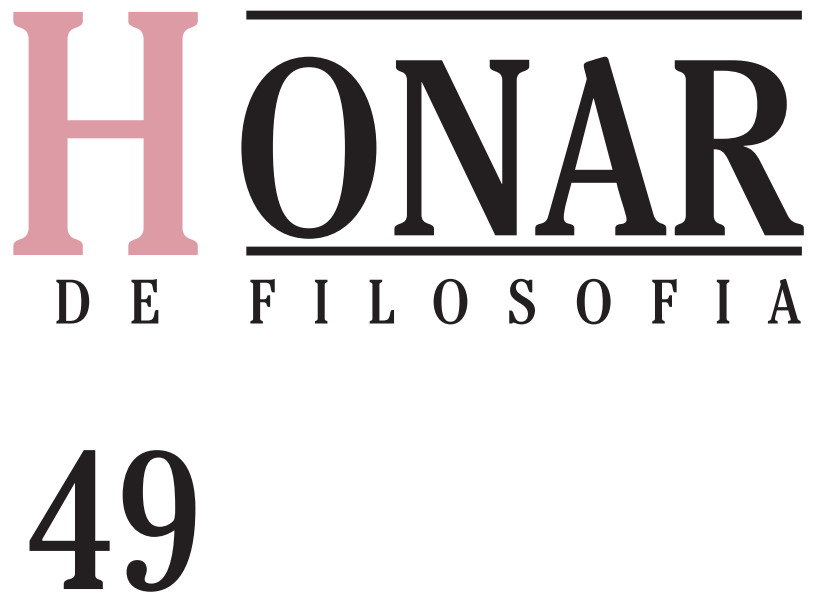

\title{
Nelson Goodman
}

Remei Capdevila Werning (coord.) 


\section{Directora}

Marta Tafalla (UAB)

Consell de redacció

Victòria Camps Cervera (UAB)

Anna Estany Profitós (UAB)

Víctor Gómez Pin (UAB)

Carl Hoefer (UAB)

David Jou Mirabent (UAB)

Joan-Carles Mèlich Sangrà (UAB)

Jaume Mensa (UAB)

Àngel Puyol (UAB)

Daniel Quesada Casajuana (UAB)

Josep M. Terricabres Noguera (UdG)

Gerard Vilar (UAB)

Consell editorial

Paula Casal (ICREA, Universitat Pompeu Fabra)

Jordi Cat (University of Indiana)

Alexander García-Düttmann (University of London)

María Herrera

(Universidad Nacional Autónoma de México)

Cristina Lafont (Northwestern University)

Christoph Menke (Goethe-Universität Frankfurt)

Carlos Moya (Universidad de Valencia)

Julián Pacho (Universidad del País Vasco)

Carlos Pereda

(Universidad Nacional Autónoma de México)

Francisca Pérez Carreño (Universidad de Murcia)

Manuel Pérez Otero (Universidad de Barcelona)

Mauricio Suárez

(Universidad Complutense de Madrid)

Carlos Thiebaut (Universidad Carlos III)

Secretari

David Hernández Abajo (UAB)
Redacció

Universitat Autònoma de Barcelona

Departament de Filosofia

08193 Bellaterra (Barcelona). Spain

Tel.: 9358116 18. Fax: 935812001

revista.enrahonar@uab.cat

Subscripció i administració

Universitat Autònoma de Barcelona

Servei de Publicacions

08193 Bellaterra (Barcelona). Spain

Tel.: 9358110 22. Fax: 935813239

sp@uab.cat

\section{Intercanvi}

Universitat Autònoma de Barcelona

Servei de Biblioteques

Secció d'Intercanvi de Publicacions

08193 Bellaterra (Barcelona). Spain

Tel.: 9358111 93. Fax: 935813219

sb.intercanvi@uab.cat

Composició

Vilanrosa

\section{Edició i impressió}

Universitat Autònoma de Barcelona

Servei de Publicacions

08193 Bellaterra (Barcelona). Spain

Tel.: 9358121 31. Fax: 935813239

sp@uab.cat

www.uab.cat/publicacions

ISSN 0211-402X

Dipòsit legal: B. 13.550-1981

Imprès a Espanya. Printed in Spain

Imprès en paper ecològic

ENRAhONAR. QuAderns de Filosofia és una revista acadèmica editada pel Departament de Filosofia de la Universitat Autònoma de Barcelona. Va ser fundada l'any $1981 \mathrm{com}$ un mitjà de difusió d'idees i debats en filosofia i altres disciplines properes. Ha estat dirigida successivament per Josep Calsamiglia, Josep Montserrat Torrents, Victòria Camps i Gerard Vilar, i actualment la dirigeix Marta Tafalla. Publica articles científics originals i també crítiques de llibres. Publica majoritàriament en català, castellà i anglès, però també en altres llengües romàniques. ENRAHONAR combina la difusió de la filosofia catalana amb l'interès per les discussions filosòfiques actuals en altres llengües i cultures.

ENRAHONAR. QUADERNS DE Filosofia té una periodicitat semestral, primavera i tardor.

Les opinions expressades en articles, notes, informacions, ressenyes i treballs publicats a ENRAHONAR són d'exclusiva responsabilitat dels seus autors.

L'acceptació d'articles es regeix pel sistema de censors. Al final del volum es poden consultar les normes del procés de selecció i les instruccions per als autors.

Bases de dades en què EnRAHONAR està referenciada

— The Philosopher's Index

- Índice Español de Ciencias Sociales y Humanidades (ISOC-CSIC)

- Répertoire Bibliographique de la Philosophie

- Revistes Catalanes amb Accés Obert (RACO)

- Latindex

- Dialnet (Unirioja)

Enrahonar es publica sota el sistema de llicències Creative Commons segons la modalitat:

Reconeixement - NoComercial (by-nc): Es permet la generació d'obres derivades sempre que no se'n faci un ús comercial. Tampoc es pot utilitzar l'obra original amb finalitats comercials. 


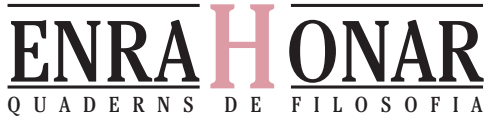 \\ Núm. 49, 2012, ISSN 0211-402X \\ http://ddd.uab.cat/record/17}

\section{Nelson Goodman}

Capdevila Werning, Remei. Presentació / Presentation.

Ammon, Sabine. Comprender el conocimiento. La contribución de Goodman al desarrollo de un concepto plural y procesal del conocimiento.

Leeten, Lars. What is "Critique of Worldmaking»? Nelson Goodman's Conception of Philosophy.

Norton-Smith, Thomas M. Ultimate Acceptability, Cultural Bias, and an American Indian World: Reflections on Nelson Goodman.

Di Scala, Sofía J. Ficción y referencia: Aportes goodmanianos para una semántica de términos ficticios.

Elgin, Catherine Z. Ejemplos elocuentes.

Jullien, Caroline. From the Languages of Art to mathematical languages, and back again.

Capdevila Werning, Remei. Construir símbolos y hacer mundos. Las dimensiones epistemológica y ontológica de la arquitectura.

Goehr, Lydia. Tres ratones ciegos: Goodman, McLuhan y Adorno sobre el arte de la música y del escuchar en la época de la transmisión global.

\section{Ressenyes}

Shottenkirk, Dena. Nominalism and Its Aftermath: The Philosophy of Nelson Goodman (Samuel Elgin).

Sartwell, Crispin. Political Aesthetics (Dialitza Colón).

Saito, Yuriko. Everyday Aesthetics (Alejandra Mizrahi).

Elkins, James; Valiavicharska, Zhivka; Kim, Alice (eds.). Art and Globalization (Loredana Niculet).

Costello, Diarmuid; Willsdon, Dominic (eds.). The Life and Death of Images (Sabina Dorneanu).

Català Domènech, Josep M. La imagen interfaz: Representación audiovisual y conocimiento en la era de la complejidad (Pol Capdevila). 


\title{
Sumari
}

Enrahonar. Quaderns de Filosofia

Núm. 49, p. 1-175, 2012, ISSN 0211-402X

Les paraules clau són en llenguatge lliure

http://ddd.uab.cat/record/17

\section{Nelson Goodman}

5-8 Capdevila Werning, Remei (Universitat Autònoma de Barcelona) Presentación / Presentation. Enrahonar, 2012, núm. 49, p. 5-8.

\author{
Abreviaturas / Abbreviations
}

11-28 Ammon, Sabine (Universität Basel)

Comprender el conocimiento. La contribución de Goodman al desarrollo de un concepto plural y procesal del conocimiento. Enrahonar, 2012, núm. 49, p. 11-28.

La definición estándar del conocimiento intenta concebir el conocimiento proposicional como una creencia verdadera y justificada $y$, con su modo de proceder, genera una serie de graves problemas. En respuesta a esto, este ensayo investiga un modelo interpretativo prometedor y alternativo del conocimiento como estructura o sistema que se acopla a las reflexiones de Nelson Goodman. A partir de una amplia base constituida por la teoría de los símbolos, es posible desarrollar un concepto de conocimiento plural y procesal, que se puede adecuar tanto a la pluralidad de distintos modos de conocimiento, como también a sus transformaciones históricas. En ello, tiene un papel decisivo la interpretación de la comprensión como un proceso constructivo que sirve como punto de partida para una descripción teórica de una dinámica transformativa. Del análisis de la relación entre procesos de comprensión y conocimiento se deriva un nuevo punto de vista que deja surgir con claridad el rasgo procesal del conocimiento y sus características específicas. Este artículo ofrece una base para una mayor diferenciación del ámbito epistémico en cuyo marco el concepto de conocimiento puede ser rehabilitado.

Palabras clave: conocimiento; comprensión; pluralismo; procesos; Goodman; equilibrio reflexivo. 
29-40 Leeten, Lars (Universität Hildesheim)

What is "Critique of Worldmaking»? Nelson Goodman's Conception of Philosophy. Enrahonar, 2012, núm. 49, p. 29-40.

The contribution examines Goodman's conception of philosophy, in particular his remark that his project can be understood as a "critique of worldmaking». It is argued that, despite dealing with epistemological questions, the general theory of symbols and worldmaking does not answer them. Rather, it can be conceived as a practical conception comparable to Kant's critique of reason or to Wittgenstein's critique of language games, i. e., as a philosophy of world orientation. It is claimed that Goodman himself could not articulate this dimension of his position appropriately as he kept using the language of epistemology. Yet many aspects of his thinking become much clearer if they are interpreted within a non-epistemological frame.

Keywords: Goodman; symbol theory; worldmaking; world orientation; world disclosure.

41-51 Norton-Smith, Thomas M. (Kent State University)

Ultimate Acceptability, Cultural Bias, and an American Indian World: Reflections on Nelson Goodman. Enrahonar, 2012, núm. 49, p. 41-51.

Nelson Goodman maintains that there is a plurality of internally consistent, equally privileged, well-made actual worlds constructed through the use of very special symbol systems — right or ultimately acceptable world versions. Using evidence from American Indian traditions, I will argue that Goodman's criteria for ultimately acceptability are culturally biased against any non-Western world version - especially a Native version. I will then offer a culturally sensitive interpretation of Goodman's criteria for ultimate acceptability that an American Indian world version satisfies, and so is numbered among the internally consistent, equally privileged, well-made actual worlds.

Keywords: American Indians; constructivism; cultural bias; Goodman; ultimate acceptability; world versions.

53-67 Di Scala, Sofía J. (Universidad de Buenos Aires)

Ficción y referencia: Aportes goodmanianos para una semántica de términos ficticios. Enrahonar, 2012, núm. 49, p. 53-67.

En los últimos años, el interés por la semántica de términos ficticios ha crecido considerablemente en el marco de la tradición analítica. De forma general, es posible distinguir dos grandes tipos de propuestas semánticas: las propuestas ontológicamente comprometidas con entidades ficticias (o realistas) y las que evitan dicho compromiso (que podríamos llamar «deflacionarias»). Entre éstas últimas, se destaca la propuesta inscripcionalista de Nelson Goodman y Catherine Elgin, que constituye el eje de análisis del presente artículo. Su objetivo principal es mostrar algunas de las ventajas de la propuesta inscripcionalista frente a las propuestas realistas. En la segunda sección, analizo algunas de las dificultades que conllevan las propuestas realistas y luego muestro que estas no tienen lugar en la propuesta de Goodman y Elgin. En la tercera sección, presento la propuesta inscripcionalista, centrándome en su ampliación, la noción de referencia y sus diversas modalidades, y también analizo una serie de enunciados problemáticos utilizando los modos referenciales anteriormente presentados.

Palabras clave: propuesta inscripcionalista; términos de ficción; Nelson Goodman. 
69-89 Elgin, Catherine Z. (Harvard University)

Ejemplos elocuentes. Enrahonar, 2012, núm. 49, p. 69-89.

Se considera que la ciencia es el espejo de la naturaleza, mientras que el arte imita la vida. De ser así, las representaciones en ambas disciplinas deberían asemejarse a sus objetos. En contra de tales teorías miméticas, argumento que la ejemplificación y no la simple semejanza es crucial. Explico en qué consiste la ejemplificación: una relación referencial de un ejemplar con alguna de sus características. Puesto que la ejemplificación es selectiva, un ejemplar puede diferir de su referente en aspectos no ejemplificados. Ésta es la razón por la cual una idealización, que estrictamente es falsa, puede ofrecer comprensión del fenómeno que le concierne. A partir de analogías con las representaciones pictóricas, muestro cómo un modelo ejemplifica características que comparte con su objeto, pone de relieve la importancia de tales características y, con ello, proporciona comprensión del sistema en cuestión.

Palabras clave: ejemplificación; representación; arte; ciencia; modelos; ficción; objetividad; comprensión; Goodman.

91-106 Jullien, Caroline (Archives Henri Poincaré. Université de Liège) From the Languages of Art to mathematical languages, and back again. Enrahonar, 2012, núm. 49, p. 91-106.

Mathematics stand in a privileged relationship with aesthetics: a relationship that follows two main directions. The first concerns the introduction of mathematical considerations into aesthetic discourse. For instance, it is common to mention the mathematical architecture of certain artistic productions. The second leads from aesthetics to mathematics. In this case, the question is that of the role and meaning that aesthetic considerations may assume in mathematics. It is indeed a widely held view among mathematicians, of whatever socio-historical context, not only to see their discipline as presenting a strong aesthetic dimension, but also to consider that this dimension plays a fundamental role in the process of developing and understanding mathematics. The main ambition of this paper is to show how Nelson Goodman's aesthetics can be used to justify this point of view and to propose a thesis concerning the aesthetic functioning of mathematics. This first result allows to resituate Goodman's aesthetics within a very classical tradition that will be described. Finally, the underlying ambition is to show the keys provided by Goodman's theory for the philosophy of mathematics.

Keywords: mathematics; aesthetics; philosophy of mathematics; Goodman; symbolization; symptoms of the aesthetic.

\section{7-120 Capdevila Werning, Remei (Universitat Autònoma de Barcelona)} Construir símbolos y hacer mundos. Las dimensiones epistemológica y ontológica de la arquitectura. Enrahonar, 2012, núm. 49, p. 107-120.

Normalmente, se supone que los edificios son entidades físicas que forman parte del mundo y, en cuanto a tales, se limitan a ser elementos materiales del mismo. Ahora bien, si, como sostiene Nelson Goodman, se considera que los edificios son también símbolos, entonces esta concepción simplificada se enriquece y la arquitectura adquiere una dimensión epistemológica con consecuencias ontológicas cruciales. Desde esta perspectiva, ya no es el material constructivo el que ayuda a construir el mundo, sino los múltiples significados de los edificios: en cuanto a símbolos, los edificios contribuyen al proceso de construcción de mundos. A partir de nociones clave de la filo- 
sofía de Goodman y mediante el análisis de varios ejemplos, el presente artículo examina de qué forma los edificios contribuyen de manera particular tanto al significado como a la creación de mundos.

Palabras clave: Goodman; arquitectura; símbolo; construcción de mundos; restauración; reconstrucción.

\section{1-154 Goehr, Lydia (Columbia University)}

Tres ratones ciegos: Goodman, McLuhan y Adorno sobre el arte de la música y del escuchar en la época de la transmisión global. Enrahonar, 2012, núm. 49, p. 121-154.

Este ensayo investiga el discurso global a la sombra de la década de 1960. Se basa en los puntos de vista de Nelson Goodman, Marshall McLuhan y Theodor W. Adorno para explorar tres conceptos centrales para la música en la época de la transmisión global: concordancia, corriente y virtualidad.

Palabras clave: Goodman; McLuhan; Adorno; transmisión; concordancia; corriente; virtualidad; música.

\section{Ressenyes}

155-159 Shottenkirk, Dena. Nominalism and Its Aftermath: The Philosophy of Nelson Goodman (Samuel Elgin).

159-161 Sartwell, Crispin. Political Aesthetics (Dialitza Colón).

162-165 Saito, Yuriko. Everyday Aesthetics (Alejandra Mizrahi).

165-167 Elkins, James; Valiavicharska, Zhivka; Kim, Alice (eds.). Art and Globalization (Loredana Niculet).

168-171 Costello, Diarmuid; Willsdon, Dominic (eds.). The Life and Death of Images (Sabina Dorneanu).

171-174 Català Domènech, Josep M. La imagen interfaz: Representación audiovisual y conocimiento en la era de la complejidad (Pol Capdevila).

175 Llibres rebuts

Normes per als col-laboradors

Normas para los colaboradores

Rules for collaborators 


\section{NORMES PER ALS COL·LABORADORS}

1. Els articles per sotmetre a consideració al consell de redacció de la revista hauran d'enviar-se per correu electrònic a l'adreça Revista.Enrahonar@uab.cat, amb l'assumpte «Article per a ENRAHONAR» i dues còpies impreses a:

Revista EnRAhonar

Departament de Filosofia

Universitat Autònoma de Barcelona

08193 Bellaterra (Barcelona). Spain

2. EnRAHONAR accepta articles escrits en qualsevol de les llengües següents: català, castellà, anglès i francès. La longitud màxima serà d'unes vint-i-cinc pàgines. Tots els articles hauran d'anar acompanyats d'un resum d'entre deu i quinze línies, i entre tres i sis paraules clau, redactats en la mateixa llengua en què estigui escrit l'article. Igualment, es lliurarà la traducció a l'anglès del títol, del resum i de les paraules clau; quan l'original sigui en anglès, es lliurarà la traducció al català o al castellà del títol, del resum i de les paraules clau. Els articles en francès hauran de presentar-se amb les traduccions a l'anglès i al català o al castellà. També seria desitjable que l'autor o l'autora deixés especificada una adreça de contacte (la de la seva universitat, per exemple) i una adreça de correu electrònic, a fi de possibilitar que els lectors i les lectores interessats puguin fer-li arribar les seves opinions i consideracions. El nom i l'adreça haurien de presentar-se a part, per garantir una revisió anònima del text.

3. Enrahonar també accepta recensions de llibres, la longitud màxima de les quals serà d'unes cinc pàgines.

4. A l'hora de fer citacions, els autors s'indicaran pel seu cognom i any de publicació, p. e.: Davidson (1984). Al final de l'article, en l'apartat de «Bibliografia», s'hi indicarà la referència completa. Els títols de llibres aniran en cursiva. Exemple:

Danto, Arthur C. (2000). The Madonna of the Future. Nova York: Farrar, Straus and Giroux.

Els articles de revista, entre cometes, i s'especificarà el títol de la revista en cursiva. Exemple:

Montserrat Torrents, Josep (1992). «L'essència del cristianisme». Enrahonar, 18, 63-69.

5. Les notes s'indicaran mitjançant números correlatius i aniran a peu de pàgina.

6. Els articles rebuts seran objecte de dos informes realitzats per avaluadors anònims i experts en la matèria, que desconeixeran la identitat de l'autor. En cas de discrepància, es recorrerà a efectuar un nou informe per part d'un tercer avaluador. En vista d'aquests informes, el consell de redacció decidirà sobre la publicació de l'article i comunicarà la decisió al seu autor.

7. Un cop acceptat l'article per ser publicat, l'autor o l'autora rebrà (si el calendari de publicacions ho permet) un joc de proves per revisar els possibles errors de la composició. També, un cop publicada la revista, l'autor o l'autora rebrà vint-i-cinc separates sense cap cost per part seva.

8. Drets de publicació

a) Enrahonar. Quaderns de Filosofia es publica sota el sistema de llicències Creative Commons segons la modalitat "Reconeixement - No Comercial (by-nc): es permet la generació d'obres derivades sempre que no se'n faci un ús comercial. Tampoc no es pot utilitzar l'obra original amb finalitats comercials». 
b) Així, quan l'autor/a envia la seva col-laboració està acceptant explícitament aquesta cessió de drets d'edició i publicació. També autoritza Enrahonar a incloure el seu treball en un fascicle de la revista per a la seva distribució i venda. Aquesta cessió sobre el treball es realitza a fi que sigui publicat a EnRAHONAR en un termini màxim de dos anys.

c) Amb l'objectiu d'afavorir la difusió del coneixement, EnRAHOnar s'adhereix al moviment de revistes d'Open Access (DOAJ), i entrega la totalitat dels seus continguts a diferents repositoris sota aquest protocol; per tant, la remissió d'un treball perquè sigui publicat a la revista pressuposa l'acceptació explícita, per part de l'autor/a, d'aquest mètode de distribució.

9. Enrahonar accepta de bon grat llibres per a la seva possible recensió. Tot llibre rebut apareixerà en una llista de «llibres arribats a la redacció». Dels més interessants (segons el parer del consell de redacció), se'n farà una recensió. ENRAHONAR no retorna els llibres rebuts.

\section{NORMAS PARA LOS COLABORADORES}

1. Los artículos para someter a consideración al consejo de redacción de la revista deberán enviarse por correo electrónico a la dirección Revista.Enrahonar@uab.cat con el asunto "Artículo para EnRAHONAR» y dos copias impresas a:

\section{Revista EnRAHONAR \\ Departament de Filosofia \\ Universitat Autònoma de Barcelona \\ 08193 Bellaterra (Barcelona). Spain}

2. Enrahonar acepta artículos escritos en cualquiera de las siguientes lenguas: catalán, castellano, inglés y francés. La longitud máxima será de unas veinticinco páginas. Todos los artículos deberán acompañarse de un resumen de entre diez y quince líneas, y entre tres y seis palabras clave, redactados en la misma lengua en que esté escrito el artículo. Igualmente, se entregará la traducción al inglés del título, del resumen y de las palabras clave; cuando el original sea en inglés, se entregará la traducción al catalán o al castellano del título, del resumen y de las palabras clave. Los artículos en francés deberán acompañarse con las traducciones al inglés y al catalán o al castellano. Asimismo, sería interesante que el autor o la autora dejaran especificada una dirección de contacto (la de su universidad, por ejemplo) y una dirección de correo electrónico para posibilitar que los lectores y las lectoras interesados puedan hacerle llegar sus opiniones y consideraciones. El nombre y la dirección deberían presentarse aparte, para garantizar una revisión objetiva del texto.

3. EnRAhonar también acepta recensiones de libros, cuya longitud máxima será de unas cinco páginas.

4. En las citas, los autores se indicarán por su apellido y año de publicación, p. e.: Davidson (1984). Al final del artículo, en el apartado de "Bibliografía», se indicará la referencia completa. Los títulos de libros irán en cursiva. Ejemplo:

Danto, Arthur C. (2000). The Madonna of the Future. Nueva York: Farrar, Straus and Giroux.

Los artículos de revista, entre comillas, y se especificará el título de la revista en cursiva. Ejemplo:

Montserrat Torrents, Josep (1992). «L'essència del cristianisme». Enrahonar, 18, 63-69. 
5. Las notas se indicarán mediante números correlativos e irán a pie de página.

6. Los artículos recibidos serán objeto de dos informes realizados por dos evaluadores anónimos y expertos en la materia, que desconocerán la identidad del autor. En caso de discrepancia, se recurrirá al juicio de un tercer evaluador. A la vista de tales informes, el consejo de redacción decidirá sobre su publicación o no, y comunicará tal decisión a los autores.

7. Una vez aceptado el artículo para su publicación, el autor o la autora recibirá (si el calendario de publicaciones lo permite) un juego de pruebas para revisar posibles errores en la composición. Asimismo, una vez publicada la revista, el autor o la autora recibirá veinticinco separatas sin ningún coste por su parte.

8. Derechos de publicación

a) Enrahonar. Quaderns de Filosofia se publica bajo el sistema de licencias Creative Commons, según la modalidad «Reconocimiento - No Comercial (by-nc): se permite la generación de obras derivadas siempre que no se haga un uso comercial. Tampoco se puede utilizar la obra original con finalidades comerciales».

b) Así, cuando el autor/a envía su colaboración está explícitamente aceptando esta cesión de derechos de edición y de publicación. Igualmente autoriza a ENRAHONAR la inclusión de su trabajo en un fascículo de la revista para su distribución y venta. Esta cesión sobre el trabajo se realiza para que sea publicado en EnRAHONAR en un plazo máximo de dos años.

c) Con el objetivo de favorecer la difusión del conocimiento, Enrahonar se adhiere al movimiento de revistas de Open Access (DOAJ), y entrega la totalidad de sus contenidos a diversos repositorios bajo este protocolo; por tanto, la remisión de un trabajo para ser publicado en la revista presupone la aceptación explícita por parte del autor/a de este método de distribución.

9. Enrahonar acepta gustosamente libros para su posible recensión. Todo libro recibido aparecerá en una lista de «libros llegados a la redacción». De los más interesantes (a juicio del consejo de redacción), se hará una recensión. Enrahonar no devuelve los libros recibidos.

\section{RULES FOR COLLABORATORS}

1. Articles to take into consideration for the editorial board should be submitted as attachment to the e-mail address Revista.Enrahonar@uab.cat with the subject «Article for ENRAHONAR», and two printed copies to:

\section{Revista EnRAHONAR \\ Departament de Filosofia \\ Universitat Autònoma de Barcelona \\ 08193 Bellaterra (Barcelona) Spain}

2. Enrahonar accepts articles written in any of these languages: Catalan, Spanish, English and French. Maximum length will be about 25 pages. All the articles will go with an abstract of 10 lines minimum and 15 maximum, and with from 3 to 6 key words, written in the same language as the article; the translation into English of title, abstract and key words will be given too. If the article is written in English, it should be given the translation into Catalan or Spanish of the title, abstract and key words. French articles will have these translations into English and Catalan or Spanish. It would be interesting as well to specify a mailing address (of the university, for example) and an e-mail address in order to make 
feasible for the readers to send back their opinions and considerations. Name and address should be given separately in order to guarantee an objective and anonymous review of the text.

3. Book reviews with no more than five pages will be taken into consideration.

4. Quotations will be made giving the author's surname and the publication year, for example: Davidson (1984). At the end of the article, in the «Bibliography», it will be given the complete reference. Book's titles will go in cursive. Example:

Danto, Arthur C. (2000). The Madonna of the Future. New York: Farrar, Straus and Giroux.

Articles will go in quotes, specifying the journal's title in cursive. Example:

Montserrat Torrents, Josep (1992). «L'essència del cristianisme». Enrahonar, 18, 63-69.

5. Footnotes should be consecutively numbered.

6. Articles will be examined by two anonymous experts in its subject, who will be unaware of the author's identity. In case of disagreement, it will be turned to a third evaluation. The editorial board will decide about the article's publication taking into account the evaluation's results and will communicate it to the author.

7. Once the article has been accepted to be published, the author will receive (if it is possible for the publication timetable) a proofs set to review possible mistakes in the layout. The author will receive as well, once the article is published, 25 reprints, free cost.

8. Publication rights

a) Enrahonar. Quaderns de Filosofia is published under the licence system Creative Commons, according to the modality «Attribution-Noncommercial (by-nc): derivative work is allowed under the condition of non making a commercial use. The original work cannot be used with commercial purposes».

b) Therefore, everyone who sends a manuscript is explicitly accepting this publication and edition cession. In the same way, he/she is authorizing ENRAHONAR. Quaderns de Filosofia to include his/her work in a journal's issue for its distribution and sale. The cession allows Enrahonar. Quaderns de Filosofia to publish the work in a maximum period of two years.

c) With the aim of favouring the diffusion of knowledge, Enrahonar. Quaderns de Filosofia joins the Open Access journal movement (DOAJ), and delivers all its contents to different repositories under this protocol; therefore, sending a manuscript to the journal also entails the explicit acceptation by its author/s of this distribution method.

9. ENRAHONAR willingly accepts books for a review. Each book received will appear in a list of «books arrived to the editorial office». To the most interesting ones (according to the editorial board judgement) will be made a review. ENRAHONAR does not return the books received. 